\title{
Use of Particle Agglutination in the Diagnosis of Dengue Virus Infection in Western Nepal
}

\author{
Ramesh Pun ${ }^{1,5}$, Krishna P. Pant ${ }^{2}$, Yogendra Shah ${ }^{5}$, Shardulendra P. Sherchand ${ }^{3}$ \\ and Basu D. Pandey ${ }^{4,5}$ \\ ${ }^{1}$ Nepal Academy of Science and Technology. ${ }^{2}$ Siddhanath Science Campus, Mahendranagar, \\ Kanchanpur. ${ }^{3}$ National College, Khusbu, Kathmandu. ${ }^{4}$ Sukraraj Tropical and Infectious \\ Diseases Hospital, Kathmandu. ${ }^{5}$ Everest International Clinic and Research Center, Kathmandu \\ e-mail: rameshpun2007@qmail.com
}

\begin{abstract}
Dengue is an emerging vector borne viral disease in Nepal. The study was conducted to detect dengue virus specific IgM and to analyze demographic distribution of positive cases. This is a cross-sectional study carried out in western region of Nepal from August-October 2007. A total of 116 serum samples were collected from suspected dengue virus infected patients visiting hospitals and investigated by particle agglutination. Out of 116 sera, 12 $(10.34 \%)$ had a positive dengue virus specific IgM. The positivity in age group below 15 years old was $23.08 \%$, $7.32 \%$ in $15-50$ years age group and $14.29 \%$ above 50 years age group. The ratio of positivity in male and female was $1: 1$. However, there were no statistical significance in the positivity with age $(\mathrm{P}=0.27)$ and sex $(\mathrm{P}=0.91)$. Thus, dengue has been established in western region of Nepal and diagnosis of the disease can be done by particle agglutination assay. The particle agglutination should be suggested for the diagnosis of dengue in all health centers of Nepal. Awareness programmes on prevention of dengue and surveillance of the disease should be conducted to reduce morbidity and mortality during possible outbreak of it.
\end{abstract}

Key words: dengue, immunoglobulin M, particle agglutination, western Nepal

\section{Introduction}

Dengue virus (DENV) causes an acute febrile illness that is transmitted from human to human by mosquitoes, predominantly Aedes aegypti. The DENV serotypes DEN-1 to DEN-4, of the genus Flavivirus, are responsible for the disease. The disease presents as dengue fever (DF) and a more severe forms dengue hemorrhagic fever (DHF) and dengue shock syndrome (DSS). Usually, DF is self limiting featured by mild to high fever, severe headache with pain behind the eyes, muscle and joint pain, rash and vomiting, however DHF resulting from secondary infection is characterized by hemorrhagic events and circulatory failure along with fever. Infection with one serotype results life long immunity but there is no cross protection against other serotypes (Gubler 1998). The disease has been a public health concern in Nepal after the first outbreak of dengue in 2006. The diagnosis of the disease is a major problem in most health centers due to poor laboratory facility. In such situation, particle agglutination (PA) is an alternative for dengue diagnosis as it does not require special laboratory set up.

The PA is merely an agglutination reaction which is performed with the help of a kit. The kit consists of IgM antibody-coated microplate and purified DENV antigen coated Ha-Hy (hydroxyapatite-coated nylon) beads. The microplate captures human IgM antibodies from the serum and addition of the beads bind to IgM molecules, which are captured on the microplate giving 
positive test. If the test sample does not contain antivirus specific antibody, the beads sediment at the bottom of the well signifying the reaction is negative. Sensitivity and specificity of the kit are $98.4 \%$ and $97.5 \%$ respectively (Yamamoto et al. 2000). We used PA assay to detect IgM antibody for the diagnosis of dengue in this study.

\section{Methodology}

This is a cross-sectional study conducted from August to November in 2007. A total number of 116 serum samples were collected by purposive sampling method during postmonsoon from Mahakali Zonal Hospital, Mahendranagar, Bheri Zonal Hospital, Nepalgunj and Lumbini Zonal Hospital, Butwal. A case was included if there was high fever with clinical symptoms and suspected of DENV infection according to WHO guidelines (WHO 1997).

The blood samples $(5 \mathrm{ml}$ from adult and $3 \mathrm{ml}$ from children) were collected from each suspected case in sterile, clean, dry and labeled test tube. The collected blood in test tube was allowed to clot at room temperature. Then, the blood was centrifuged at 5000 rpm for 5 minutes and the serum was separated. The serum was then collected in vial and stored at $4^{\circ} \mathrm{C}$ for a week. The samples were transported to Everest International Clinic and Research Center (EICRC), Kathmandu maintaining reverse cold chain. Aliquots were made for analysis and refrigerated at $2-8^{\circ} \mathrm{C}$ until tested.

Sera were tested for anti-dengue IgM with PA assay kit (Pentax, Japan). The diluted serum (1:100) was added to the wells and incubated for 30 minutes at room temperature $\left(20-25^{\circ} \mathrm{C}\right)$. After washing the wells, dengue virus antigen-coated beads slurry was added and allowed to settle for an hour. The result was interpreted on the basis of beads agglutination pattern (Fig. 1).

Patients' personal details about the symptoms and age, sex, etc were obtained through a questionnaire method by direct interview after obtaining the consent. Data analysis was done with the help of chi square test using Win pepi software.

\section{Result and Discussion}

A total of 116 study subjects were included in the research for detection of anti-dengue IgM by PA (Table 1). Out of the total subjects, $12(10.34 \%)$ had positive anti-dengue IgM test. The number of positive dengue serology for males and females were six each (Fig. 2). The percentage of positive cases were 23.08 (3/13) in the age group below 15 years old. Similarly, $7.32 \%$ (6/ 82) of positive cases were from 15- 50 years old and $14.29 \%(3 / 21)$ from above 50 years old (Fig.3). The association of DENV infection with sex $(\mathrm{P}=0.91)$ and age $(\mathrm{P}=0.27)$ was not statistically significant.

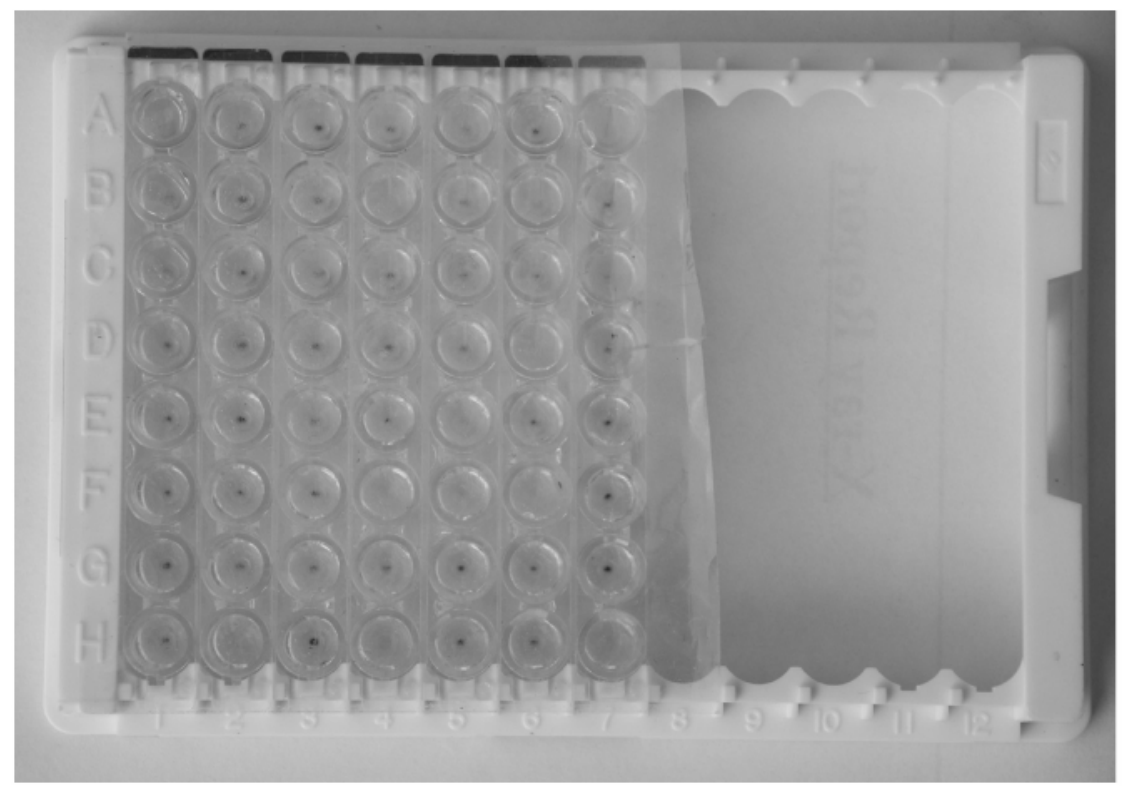

Fig. 1. Image showing beads agglutination pattern 
Table 1. Distribution of the subjects by age and sex

\begin{tabular}{l|r}
\hline Samples collected & 116 \\
Male: female ratio & $1.07: 1(60: 56)$ \\
Age (years) & 13 \\
$<15$ (child) & 82 \\
$15-50$ (adult) & 21 \\
$>50$ (old) & \\
\hline
\end{tabular}

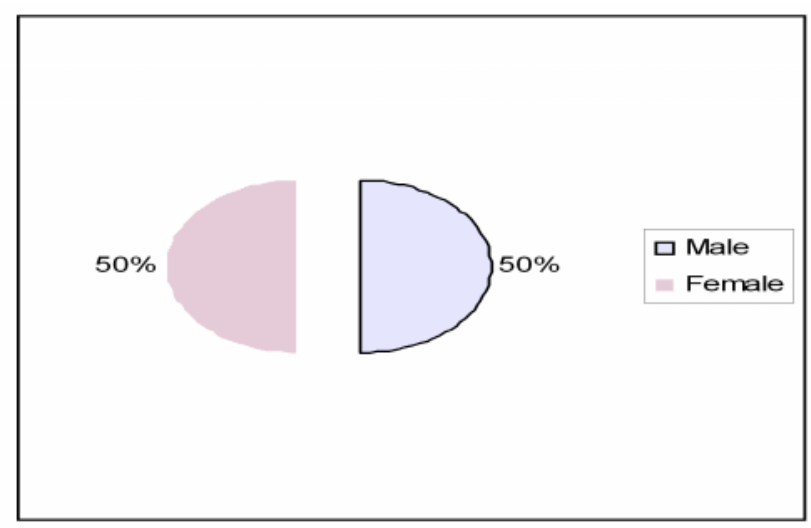

Fig. 2. Sexwise distribution of patients with anti-dengue IgM

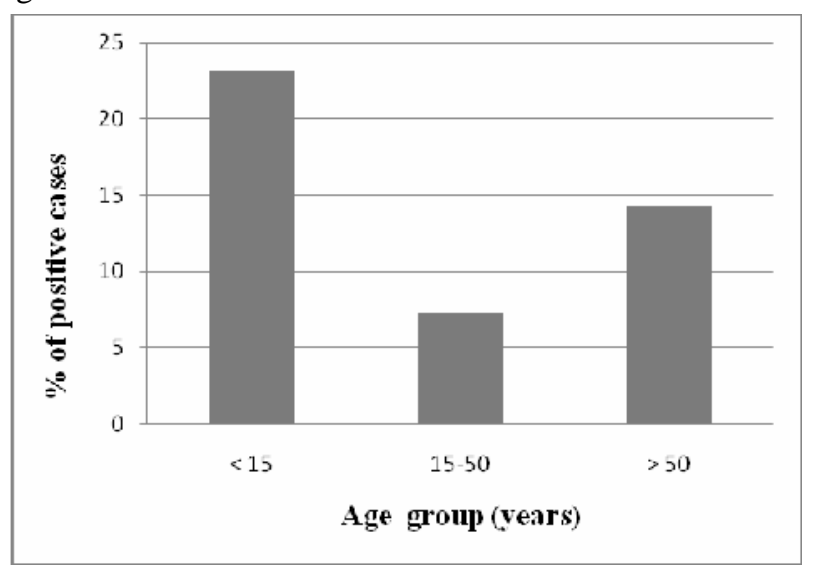

Fig. 3. Agewise distrbution of patients with antidengue IgM

The exact year about the introduction of dengue in Nepal is not clear, but sporadic cases were reported in foreigners visiting Nepal in the late 1980s and 1990s (Kurane et al. 2000). Since then, the first case of DF was reported in the country in a Japanese volunteer in the year 2004 (Pandey et al. 2004). The outbreak of DF was documented in November 2006 in several locations of Terai region of Nepal followed by 2010 epidemic, which claimed hundreds of hospitalization and few deaths (STIDH 2010). All the serotypes of DENV have been found in Nepal indicating the possibility of severe form of the disease (WHO/SEARO 2006). Dengue is a big threat for health as the incidence of the disease has increased 30-fold with increasing geographic expansion to new countries as well as from urban to rural settings (WHO 2008).

The anti-dengue IgM positivity was $10.34 \%$ in western region. The result was similar to the study conducted by Sherchand et al. (2001). The positivity to dengue indicates the circulation of DENV in the region. All the study areas are close to the borders of India, where dengue is endemic. The movement of people and the use of modern transportation across the border increase the transmission of the disease. The rate of transmission is pronounced in post monsoon period as the distribution of Ae. aegypti larval indices is highest during this period (Chakravorti \& Kumaria 2005). Heavy rainfall results decrease in temperature and subsequently relative humidity is increased. This favorable situation along with abundant stocks of fresh water reservoir generated due to rain, develop optimum conditions for mass breeding and propagation of vector as well as transmission of the virus.

The dengue positive cases were clustered in the age group below 15 years old. The result was not in accordance with the data obtained during first outbreak of dengue in Nepal (WHO/SEARO 2006) and the study carried out by Gupta et al. (2006). This might be due to exposure of children to DENV as a result of day time transmission in the schools and outdoor activities of the children. The discrepancy of positive cases in different age groups might be due to disproportionate collection of samples in each age group. However, age was an independent predictor for DENV infection $(\mathrm{P}>0.05)$.

Out of 12 positive cases, male and female patients were six each comprising $50 \%$ of the total positive cases. The finding was similar to the result obtained by Dominguiz (1997), where $51 \%$ were males and $47 \%$ were females infected with DENV. However, the association between sex and the disease was not statistically significant $(\mathrm{P}>0.05)$. 
This study was based on PA test, which also detects cross reacting antibodies to other flavivirus such as Japanese encephalitis (JE) virus. However, subjects with previous JE immunizations were excluded from the study. Therefore, false positive results because of cross reactivity were minimal.

The diagnosis of dengue can be done by culture and molecular techniques but, these methods are not feasible in health centers of Nepal. The isolation of DENV in cell culture and detection of viral RNA by reverse transcriptase-polymerase chain reaction is not possible in routine laboratories because of long reaction time and high costs (Kittigul \& Suankeow 2002). Enzyme linked immunosorbent assay (ELISA) has been widely used for the diagnosis of DENV infection but this method is also expensive and takes several hours to complete (Lam \& Devine 1998). Therefore, PA could be the choice for dengue diagnosis because it is easy to perform and relatively inexpensive as compared to ELISA. Moreover, the result can be interpreted in a fairly short time. The reliability of PA has been trusted as it has sensitivity of $98 \%$ and specificity of $96 \%$ whereas positive prediction value of $90 \%$ and $99 \%$ negative prediction value during evaluation of the assay with gold standard ELISA (Sah et al. 2009).

Clearly, dengue was evident in western region of Nepal and PA was used as a serological tool to detect IgM antibody for the diagnosis of DENV infection. The PA should be recommended in all health centers for dengue diagnosis in developing countries like Nepal. Besides, awareness programmes on prevention of dengue and surveillance of the disease should be conducted to reduce morbidity and mortality during possible outbreak of dengue.

\section{Acknowledgements}

We thank Everest International Clinic and Research Center, Kathmandu, Kiran Pandey and Mahmood Ahamad Siddiqi for their help and cooperation.

\section{References}

Chakravorti, A. and R. Kumaria. 2005. Eco-epidemiological analysis of dengue infection during an outbreak of dengue fever, India. Journal of Virology 2: 1-6.
Dominguez, N.N. 1997. Current DF/DHF prevention and control programme in the Philippines. Dengue Bull/WHO.

Gubler, D.J. 1998. Dengue and dengue hemorrhagic fever. Journal of Clinical Microbiology and Revolution 11: 480496.

Gupta, B., D. Lalit, K. Geetanjali and B. Shobha. 2006. The changing epidemiology of dengue in Delhi, India. Journal of Virology 3: 92.

Kittigul, L. and K. Suankeow. 2002. Use of rapid immunochromatographic test for early diagnosis of dengue virus infection. European Journal of Clinical Microbiology and Infectious Diseases 21: 224-226.

Kurane, I., T. Takasaki and K. Yamada. 2000. Trends in flavivirus infections in Japan. Journal of Emerging Infectious Diseases 6: 1-3.

Lam, S.K. and P.L. Devine. 1998. Evaluation of capture ELISA and rapid immunochromatographic test for the determination of IgM and IgG antibodies produced during dengue infection. Journal of Clinical Diagnosis and Virology 10: 75-81.

Pandey, B.D., S.K. Rai, K. Morita and I. Kurane. 2004. First case of dengue in Nepal. Journal of Nepal Medical College 6: 157-159.

STIDH. 2010. Report on dengue fever. Clinical Research Unit, Sukraraj Tropical and Infectious Diseases Hospital, Kathamndu, Nepal.

Sah, O.P., S. Subedi, K. Morita and B. D. Pandey. 2009. Serodiagnosis of dengue by particle agglutination assay. Journal of Nepal Health Research Council 7: 29-32.

Sherchand, B., B.D. Pandey, K. Haruki, M. Jimba. 2001. Sero-diagnosis of Japanese encephalitis and dengue virus infection from clinically suspected patients of Nepal.Journal of Institute of Medicine 23: 81-85.

WHO/SEARO. 2006. Outbreak investigation of dengue in Nepal.

WHO. 1997. Dengue hemorrhagic fever: diagnosis, treatment and control. 2nd ed.

WHO. 2008. Dengue and dengue haemorrhagic fever. Factsheet No. 117, revised. Geneva,World Health Organization (http://www.who.int/mediacentre/ factsheets/fs117/en/).

Yamamoto, A., M. Nakayama, M. Tashiro, T. Ogawa and I. Kurane. 2000. Hydroxyapatite-coated nylon beads as a new reagent to develop a particle agglutination assay system for detecting Japanese encephalitis virus-specific human antibodies. Journal of clinical virology 19: 195-204. 